\title{
Prevalence of Chronic Metoclopramide Use and Associated Diagnoses in the US Pediatric Population
}

\author{
Rachel B. Weinstein ${ }^{1} \cdot$ Daniel Fife $^{1} \cdot$ Sheldon Sloan $^{2} \cdot$ Erica A. Voss $^{1} \cdot$ \\ William Treem ${ }^{3}$
}

Published online: 27 May 2015

(c) The Author(s) 2015. This article is published with open access at Springerlink.com

\begin{abstract}
Objective Metoclopramide is the only medication widely used to promote gastrointestinal motility in the USA. Despite its appreciable risk of central nervous system complications, it continues to be prescribed to children for chronic use. We sought to estimate the prevalence of chronic metoclopramide use among US children and identify the diagnoses that may have prompted this use. The US metoclopramide label lists only two indications in adults: symptomatic gastroesophageal reflux (GERD) and diabetic gastroparesis. The latter is rare in children so, in examining the indications likely to have prompted chronic metoclopramide use, we focused on GERD.

Methods From two health services databases representing privately and publically insured children, respectively, we estimated the number of US children who used metoclopramide chronically and identified the diagnoses recorded at approximately the time when the chronic use began. We defined chronic use liberally as $\geq 35$ days' supply, or conservatively as $\geq 130$ days' supply in a 6-month period.
\end{abstract}

Electronic supplementary material The online version of this article (doi:10.1007/s40272-015-0136-2) contains supplementary material, which is available to authorized users.

Rachel B. Weinstein

rweinst1@its.jnj.com

1 Epidemiology, Janssen Research and Development LLC, 1125 Trenton-Harbourton Rd, Titusville, NJ 08560, USA

2 Global Medical Affairs, Janssen Research and Development LLC, 200 Tournament Drive, Horsham, PA 19044, USA

3 Global Medical Organization, Child Health Innovation and Leadership Department (CHILD), Janssen Research and Development, LLC, 920 US Route 202, Raritan, NJ 08869, USA
For each chronic-use definition, insurance type, and age group, we estimated the proportion of children using metoclopramide chronically. We applied these proportions to US population estimates.

Results Under the liberal and conservative definitions, respectively, 89,020 and 28,222 US children used metoclopramide chronically.

Conclusion In spite of its risk, substantial numbers of US children use metoclopramide chronically for symptoms suggestive of GERD.

\section{Key Points}

Metoclopramide, the only gastrointestinal motility agent widely used in the USA, has a black-box warning for tardive dyskinesia.

We found that, nonetheless, it is used chronically by at least 28,000 US children, most of whom have at least one health service claim for symptomatic gastroesophageal reflux (GERD).

This suggests a perception that many US children with symptoms suggestive of GERD are not adequately treated by lifestyle changes and acid suppression.

\section{Introduction}

Metoclopramide, the only gastrointestinal (GI) motility agent that is widely used in the USA, crosses the blood-brain barrier and is associated with central nervous system (CNS) side effects, including irritability, tardive dyskinesia, and 
lowering of the seizure threshold [1]. Its label includes a black-box warning for tardive dyskinesia and a statement that extrapyramidal symptoms occur more frequently in pediatric patients and adult patients less than 30 years of age. Two clinical reviews on metoclopramide treatment of gastroparesis report tardive dyskinesia in 1-15\% of treated patients, although there is no prospective data on this risk [2, 3]. This suggests that prescription of metoclopramide to children for chronic use reflects a perception of a substantial need that is not met by other medications available in the USA. We sought to estimate the prevalence of chronic metoclopramide use among US children and identify the diagnoses that may have prompted its use.

The prescribing information in the US Package Insert (USPI) for oral metoclopramide lists two indications for adults only: symptomatic gastroesophageal reflux (GERD) and diabetic gastroparesis $[4,5]$. Because the latter is rare among children, we focused on the former. The prevalence of refractory GERD in the pediatric population is suspected to be low, but is not well documented, and for those affected, the need for effective chronic treatment is great. Therefore it is of interest to have a better understanding of the prevalence of this condition among children.

A diagnosis of GERD recorded in a claims database does not mean the patient met the formal criteria for the diagnosis. He or she may simply have had symptoms suggestive of the diagnosis. However, the recording of the diagnosis in the database, together with the prescription of metoclopramide for chronic use and the concurrent prescription of an acid suppressing medication, strongly suggests the prescribing physician found chronic GERD to be a credible cause of the patient's symptoms and found those symptoms to be unresponsive to more conservative treatment, including changes in feeding and positioning, and the use of acid suppressive medications only [6, 7]. This study directly estimates the prevalence of chronic metoclopramide use in US children under either of two definitions of chronic use.

Furthermore, refractory GERD in children can be defined as the persistence of symptoms and esophageal inflammation beyond 1 year of age in spite of the chronic application of accepted feeding and lifestyle changes and medical intervention with the standard of care [acid suppression with histamine 2 receptor antagonists (H2RAs) and/or proton pump inhibitors (PPIs)]. In this study, the number of children aged $>1$ year who used metoclopramide chronically and had a diagnosis of GERD at about the time they began its chronic use approximates, but does not strictly meet, these criteria.

Refractory GERD has a markedly increased prevalence among children who have certain underlying conditions, including CNS dysfunction; congenital genetic/metabolic conditions, for example, Down syndrome, Rett syndrome,
Cornelia de Lange syndrome; chronic pulmonary disease such as cystic fibrosis and chronic asthma; and repaired esophageal atresia, trachea-esophageal fistula or congenital diaphragmatic hernia [8]. Especially among those with severe CNS dysfunction, motility abnormalities often persist even after treatment of GERD with acid suppressive agents [9]. Because the prescription of metoclopramide for chronic use in such patients seemed likely to reflect symptoms similar to those of GERD, even if the diagnosis of GERD was not entered into the claims database, we tabulated separately the numbers of children in the database who used metoclopramide chronically, had a diagnosis suggestive of an underlying condition with a high risk of GERD, but did not have a diagnosis of GERD, at approximately the time they began chronic use of metoclopramide.

\section{Methods}

We used two large healthcare claims databases [Truven Health MarketScan ${ }^{\circledR}$ Multi-State Medicaid and Truven Health MarketScan ${ }^{\circledR}$ Commercial Claims and Encounters (CCAE) databases]. The CCAE is representative of a privately insured population and the Medicaid database contains data on Medicaid enrollees from multiple states, but not all states. The version of CCAE we used for this study described over 90 million members with medical and pharmacy coverage. The Medicaid data reflected over 9 million members enrolled between January 2006 and January 2009 in states that shared these data with Truven Health Analytics. The mean age in the CCAE population was 31.3 years; $49 \%$ of the population was male; and the average duration of observation in the database was 2.3 years. The mean age in the Medicaid population was 17.0 years; $42 \%$ of the population was male; and the average duration of observation in the database was 1.5 years. These databases capture diagnoses and procedures submitted for office visits and hospitalizations and also capture prescription drug dispensing in retail and mailorder pharmacies. The New England Institutional Review Board (NEIRB\# 12-284) has determined that studies conducted in the databases used in this study do not meet the definition of human subjects research.

\subsection{Identifying the Cohort}

We first identified a cohort of patients who were either continuously enrolled in each database for the 2-year period from July 1, 2007 to June 30, 2009 (except for gaps of less than 32 days) or, for those born during July 2007 through to the end of December 2008, were enrolled since birth. This defines the population in which our study is nested. Cohort 
members were children $\leq 17$ years old during the period January 1, 2008-December 31, 2008. Within this group, we identified the subjects with chronic metoclopramide use, defined the index date for each such subject as the date of the first metoclopramide dispensing in 2008, and looked for diagnoses near the index date that could be reasons for that subject's chronic metoclopramide use.

\subsection{Identifying Metoclopramide Users}

The observation window for metoclopramide use began with the date of the first metoclopramide dispensing in 2008 and continued for 6 months (183 days). Users were defined as anyone with at least one dispensing of a metoclopramide preparation for oral use in 2008. Two alternative definitions of chronic user were based on the number of dispensings and days' supply dispensed, starting with, and including, the first metoclopramide dispensing to each child in 2008. Requiring a minimum of two dispensings of metoclopramide increases the likelihood that at least one of those dispensings was consumed:

1. More restrictive definition Chronic use required at least two dispensings and a minimum of 130 days' supply in the 183 days from the initial dispensing.

2. Less restrictive definition Chronic use required at least two dispensings and a minimum of 35 days' supply in the 183 days from the initial dispensing.

Because dispensings with $>90$ days' supply were not considered plausible, only dispensings with 90 days' supply or less were included. If there were multiple dispensings of metoclopramide on the same day, only the largest days' supply was included in the total days' supply. This rule was applied because patients could get multiple prescriptions of different dose sizes to add up to the necessary dose. Finally, any days' supply that would have been taken after the patient's 183-day follow-up period was censored (e.g., if a patient received a prescription on March 12, 2008 for 30 days' supply, but the end of their follow-up was on March 13, 2008, the days after March 13 would not be included in their total days' supply of metoclopramide).

\subsection{Identifying Diagnoses}

For all cohort members, GI-related diagnoses were obtained by searching the time period from 183 days prior to the index date through to 183 days following the index date. For cohort members with less than the full 183-day lead-in period (i.e., for those who were aged under 2 years), the search window started with the enrollment date and ended 183 days after the index date.
To better understand the characteristics of the population of pediatric metoclopramide users, we defined broad GI-related categories that include diagnoses for which metoclopramide might be used. The three broad but nonoverlapping diagnosis categories were (1) a diagnosis of GERD; (2) GI diagnoses other than GERD, but possibly related to GERD; and (3) neurogastroenterology (neuroGI) diagnoses of conditions with a known increased risk of chronic GERD. The International Classification of Diseases, Ninth Revision (ICD-9) diagnoses codes in each category are listed in Online Resource Table A1. GERD was defined by having a diagnostic code for either reflux esophagitis or esophageal reflux. Common examples for "GI diagnoses" other than GERD, but possibly related to GERD, include gastroparesis, vomiting, asthma, chest pain, pneumonia and cystic fibrosis. The "neuro-GI diagnoses" were neurological or CNS conditions that could be accompanied by GI comorbidities for which metoclopramide may be prescribed, such as cerebral palsy, Down syndrome, and epilepsy. Although the categories did not overlap in the sense that no diagnosis appeared in more than one category, it was possible for a patient with multiple diagnoses to appear in more than one category.

\subsection{Estimating the Number of Chronic Metoclopramide Users in the US Pediatric Population}

Assuming the databases are representative of privately and Medicaid insured people in the USA, and that all children are covered by private insurance or Medicaid eligible, the rates in the databases could be applied to estimates of the corresponding Medicaid and privately insured populations to generate estimates for overall chronic metoclopramide use. Estimates for the number of privately insured children aged 17 or less in the USA were obtained by subtracting the number of Medicaid eligible in the USA in 2008 [10] from US census population counts [11] for that year (see Online Resource Tables A2 and A3). Within each age group, $<1,1,2-6$, and 7-17 years, the proportion of children in each database who were chronic users was then applied to the corresponding privately insured and Medicaid populations. To confirm that we had identified the population of interest for our study with symptoms suggestive of GERD, we also examined the use of H2RAs and PPIs during the follow-up period. Specifically, we identified the number and proportion of children who took metoclopramide chronically and had a dispensing of either an H2RA drug or a PPI that overlapped with the metoclopramide dispensing on the index date and/or the 183-day follow-up. 


\section{Results}

Table 1 shows how the criteria applied to each database resulted in the cohorts of privately and publically insured patients, respectively. In the private insured database, there were 7,086,142 children aged $\leq 17$ during the study period and $3,076,597$ met the study entry criteria of continuous enrollment during July 1, 2007 through to June 30, 2009, except for gaps of less than 32 days. Similarly, in the
Medicaid database there were $3,119,150$ children aged $\leq 17$ during the study period and 1,060,030 met the study entry criteria of continuous enrollment during July 1, 2007 through to June 30, 2009, except for gaps of less than 32 days. In each age group, less than $2 \%$ were metoclopramide users and less than $1 \%$ were chronic metoclopramide users.

Tables 2 and 3 give the percentage of the study cohorts with a diagnosis of GERD, with broadly related GI diagnoses

Table 1 Identification of eligible patients in the Truven Health MarketScan ${ }^{\circledR}$ Commercial Claims and Encounters (CCAE) and Multi-State Medicaid databases

\begin{tabular}{lcc}
\hline & CCAE $(n)$ & Medicaid $(n)$ \\
\hline All children aged 0-17 during July 1, 2007-June 30, 2009 & $7,086,142$ & $3,119,150$ \\
Study entry criteria: children continuously enrolled (except for gaps $<32$ days) & $3,076,597$ & $1,060,030$ \\
from July 1, 2007 to June 30, 2009, or since birth for those born during & \\
July 2007 through to the end of December 2008 & 7128 \\
No. with at least 1 metoclopramide dispensing during 2008 and days' supply $\leq 90$ & 2757 \\
No. with at least 2 metoclopramide dispensings & 2295 \\
Chronic use (unrestrictive): no. with 35 days' supply & 668 \\
Chronic use (restrictive): no. with 130 days' supply & 7126 \\
\hline
\end{tabular}

Table 2 Number of children with GI-related ${ }^{\mathrm{a}}$ diagnoses among chronic users of metoclopramide, Truven Health MarketScan ${ }^{\circledR}$ Commercial Claims and Encounters database

\begin{tabular}{|c|c|c|c|c|c|}
\hline Diagnosis & Total cohort & Non-users & Users $^{\mathrm{b}}$ & Chronic $(35 \text { days })^{\mathrm{c}}$ & Chronic (130 days) \\
\hline \multicolumn{6}{|l|}{ Age $<1$ year } \\
\hline Total cohort size & $192,560(100 \%)$ & $190,597(100 \%)$ & $1963(100 \%)$ & $900(100 \%)$ & $226(100 \%)$ \\
\hline GERD & $22,002(11.43 \%)$ & $20,478(10.74 \%)$ & $1524(77.64 \%)$ & $729(81.00 \%)$ & $190(84.07 \%)$ \\
\hline GI diagnoses & $16,487(8.56 \%)$ & $15,788(8.28 \%)$ & $699(35.61 \%)$ & $319(35.44 \%)$ & $80(35.4 \%)$ \\
\hline Neuro-GI disorders & $34,921(18.14 \%)$ & $34,242(17.97 \%)$ & $679(34.59 \%)$ & $340(37.78 \%)$ & $99(43.81 \%)$ \\
\hline \multicolumn{6}{|l|}{ Age 1 to $<2$ years } \\
\hline Total cohort size & $220,597(100 \%)$ & $219,223(100 \%)$ & $1374(100 \%)$ & $593(100 \%)$ & $178(100 \%)$ \\
\hline GERD & $9393(4.26 \%)$ & $8545(3.90 \%)$ & $848(61.72 \%)$ & $466(78.58 \%)$ & $142(79.78 \%)$ \\
\hline GI diagnoses & $22,539(10.22 \%)$ & $21,995(10.03 \%)$ & $544(39.59 \%)$ & $237(39.97 \%)$ & $74(41.57 \%)$ \\
\hline Neuro-GI disorders & $14,504(6.57 \%)$ & $14,106(6.43 \%)$ & $398(28.97 \%)$ & $228(38.45 \%)$ & $81(45.51 \%)$ \\
\hline \multicolumn{6}{|l|}{ Age 2-6 years } \\
\hline Total cohort size & $689,070(100 \%)$ & $688,227(100 \%)$ & $843(100 \%)$ & $248(100 \%)$ & $108(100 \%)$ \\
\hline GERD & $6035(0.88 \%)$ & $5745(0.83 \%)$ & $290(34.4 \%)$ & $162(65.32 \%)$ & $76(70.37 \%)$ \\
\hline GI diagnoses & $55,668(8.08 \%)$ & $55,283(8.03 \%)$ & $385(45.67 \%)$ & $140(56.45 \%)$ & $63(58.33 \%)$ \\
\hline Neuro-GI disorders & $17,967(2.61 \%)$ & $17,780(2.58 \%)$ & $187(22.18 \%)$ & $123(49.6 \%)$ & $69(63.89 \%)$ \\
\hline \multicolumn{6}{|l|}{ Age 7-17 years } \\
\hline Total cohort size & $1,974,370(100 \%)$ & $1,971,422(100 \%)$ & $2948(100 \%)$ & $554(100 \%)$ & $156(100 \%)$ \\
\hline GERD & $17,165(0.87 \%)$ & $16,420(0.83 \%)$ & $745(25.27 \%)$ & $287(51.81 \%)$ & $82(52.56 \%)$ \\
\hline GI diagnoses & $76,965(3.9 \%)$ & 75,707 (3.84 \%) & $1258(42.67 \%)$ & $305(55.05 \%)$ & $82(52.56 \%)$ \\
\hline Neuro-GI disorders & $39,536(2.00 \%)$ & $39,119(1.98 \%)$ & $417(14.15 \%)$ & $158(28.52 \%)$ & $64(41.03 \%)$ \\
\hline
\end{tabular}

GERD symptomatic gastroesophageal reflux, GI gastrointestinal, Neuro-GI neurogastroenterology

a Note that patients could be assigned to multiple diagnostic groups

b The last three columns are nested. Users includes all chronic users

' Chronic users with 130 days' supply were included in with the chronic users with at least 35 days' supply 
Table 3 Number of children with GI-related diagnoses ${ }^{\mathrm{a}}$ among chronic users of metoclopramide, Truven Health MarketScan ${ }^{\circledR}$ Multi-State Medicaid database

\begin{tabular}{|c|c|c|c|c|c|}
\hline Diagnosis & Total cohort & Non-users & Users $^{\mathrm{b}}$ & Chronic $(35 \text { days })^{\mathrm{c}}$ & Chronic (130 days) \\
\hline \multicolumn{6}{|l|}{ Age $<1$ year } \\
\hline Total cohort size & $180,277(100 \%)$ & $177,100(100 \%)$ & $3177(100 \%)$ & $1356(100 \%)$ & $267(100 \%)$ \\
\hline GERD & $26,014(14.43 \%)$ & $23,531(13.29 \%)$ & $2483(78.16 \%)$ & $1180(87.02 \%)$ & $233(87.27 \%)$ \\
\hline GI diagnoses & $29,108(16.15 \%)$ & $27,717(15.65 \%)$ & $1391(43.78 \%)$ & $646(47.64 \%)$ & $124(46.44 \%)$ \\
\hline Neuro-GI disorders & $32,157(17.84 \%)$ & $31,026(17.52 \%)$ & $1131(35.6 \%)$ & $575(42.4 \%)$ & $125(46.82 \%)$ \\
\hline \multicolumn{6}{|l|}{ Age 1 to $<2$ years } \\
\hline Total cohort size & $130,976(100 \%)$ & $129,747(100 \%)$ & $1229(100 \%)$ & $531(100 \%)$ & $140(100 \%)$ \\
\hline GERD & $6,898(5.27 \%)$ & $6072(4.68 \%)$ & $826(67.21 \%)$ & $446(83.99 \%)$ & $117(83.57 \%)$ \\
\hline GI diagnoses & $24,088(18.39 \%)$ & $23,405(18.04 \%)$ & $683(55.57 \%)$ & $306(57.63 \%)$ & $86(61.43 \%)$ \\
\hline Neuro-GI disorders & $11,931(9.11 \%)$ & $11,413(8.8 \%)$ & $518(42.15 \%)$ & $306(57.63 \%)$ & $104(74.29 \%)$ \\
\hline \multicolumn{6}{|l|}{ Age 2-6 years } \\
\hline Total cohort size & $262,939(100 \%)$ & $262,057(100 \%)$ & $882(100 \%)$ & $452(100 \%)$ & $186(100 \%)$ \\
\hline GERD & $4562(1.74 \%)$ & $4089(1.56 \%)$ & $473(53.63 \%)$ & $325(71.9 \%)$ & $128(68.82 \%)$ \\
\hline GI diagnoses & $35,928(13.66 \%)$ & $35,421(13.52 \%)$ & $507(57.48 \%)$ & $267(59.07 \%)$ & $107(57.53 \%)$ \\
\hline Neuro-GI disorders & $20,684(7.87 \%)$ & $20,203(7.71 \%)$ & $481(54.54 \%)$ & $338(74.78 \%)$ & $160(86.02 \%)$ \\
\hline \multicolumn{6}{|l|}{ Age $7-17$ years } \\
\hline Total cohort size & $485,838(100 \%)$ & $484,000(100 \%)$ & $1838(100 \%)$ & $647(100 \%)$ & $255(100 \%)$ \\
\hline GERD & $8648(1.78 \%)$ & $8038(1.66 \%)$ & $610(33.19 \%)$ & $371(57.34 \%)$ & $148(58.04 \%)$ \\
\hline GI diagnoses & $40,007(8.23 \%)$ & $39,118(8.08 \%)$ & $889(48.37 \%)$ & $327(50.54 \%)$ & $120(47.06 \%)$ \\
\hline Neuro-GI disorders & $34,670(7.14 \%)$ & $34,093(7.04 \%)$ & $577(31.39 \%)$ & $382(59.04 \%)$ & $192(75.29 \%)$ \\
\hline
\end{tabular}

GERD symptomatic gastroesophageal reflux, GI gastrointestinal, Neuro-GI neurogastroenterology

a Note that patients can be assigned to multiple diagnostic groups

b The last three columns are nested. Users includes all chronic users

' Chronic users with 130 days' supply were included in with the chronic users with at least 35 days' supply

not including GERD, and with neuro-GI diagnoses not including GERD, and for each age group for the CCAE and Medicaid database populations, respectively. In each database and for either definition of chronic use, 2-4\% of chronic metoclopramide users carried a diagnosis of gastroparesis (numbers not shown). Reading across rows in both tables, the percentages with diagnoses were higher among metoclopramide users compared with non-users, and highest amongst those with chronic metoclopramide use, as would be expected. Among privately insured and Medicaid-insured subjects, the proportion of chronic users who had a diagnosis of GERD was approximately $80 \%$ in those $<2$ years of age, $60-70 \%$ in those aged 2-6 years, and $50 \%$ in those aged 7-17 years. For all ages combined, 70-75\% of chronic users had a diagnosis of GERD (numbers not shown). As age increased, the percentage of chronic users with a diagnosis of GERD decreased and the percentage with other GI-related diagnoses increased. Because the number of chronic users of metoclopramide per year of age decreased substantially with age (e.g., the number per year of age in age group 2-6 years is the number in the table divided by 5), the number of children per year of age who had other GI diagnoses did not increase with age.
Substantial proportions of chronic users (approximately 40-60 \%) had broad GI-related or neuro-GI diagnoses in each age group in both the Medicaid and privately insured populations (Tables 2 and 3). In the Medicaid population, the percentage of chronic users with neuro-GI conditions was substantially higher among those aged $>1$ year than among those aged $\leq 1$ year. Additionally, over $87 \%$ of chronic users (either type) who were privately insured had at least one of the three types of GI diagnoses in the tables (numbers not shown). For Medicaid insured children, over $90 \%$ of chronic metoclopramide users had at least one of these GI diagnosis types.

We also examined the extent of concomitant use of H2RAs and PPIs with metoclopramide. Concomitant use was defined as an overlap of at least 1 day based on dispensing date and days' supply. Regardless of diagnostic category, more than $60 \%$ of children who took metoclopramide chronically took concomitantly a medication that suppressed gastric acid. Among those with a diagnosis of GERD and the less restrictive definition of chronic metoclopramide use, over $85 \%$ also had concomitant dispensing of a gastric acid suppressor in both databases (data not shown). And for those with a diagnosis of GERD and the 
Table 4 Estimated US prevalence ${ }^{\mathrm{a}}$ of pediatric population with chronic metoclopramide use, by insurance type and age group

\begin{tabular}{|c|c|c|c|c|c|c|c|c|c|c|}
\hline & \multicolumn{5}{|c|}{ Chronic use ( $\geq 35$ days): age group } & \multicolumn{5}{|c|}{ Chronic use ( $\geq 130$ days): age group } \\
\hline & $<1$ year & 1 year & $2-6$ years & $7-17$ years & $\begin{array}{l}\text { Total: } \\
\text { age } \\
0-17\end{array}$ & $<1$ year & 1 year & $2-6$ years & $7-17$ years & $\begin{array}{l}\text { Total: } \\
\text { age } \\
0-17\end{array}$ \\
\hline \multicolumn{11}{|l|}{ Medicaid } \\
\hline 2008 population & $2,415,672$ & $2,064,288$ & $9,523,468$ & $15,188,287$ & & $2,415,672$ & $2,064,288$ & $9,523,468$ & $15,188,287$ & \\
\hline $\begin{array}{l}\text { Chronic use } \\
\text { (\% of population) }\end{array}$ & 0.75 & 0.41 & 0.17 & 0.13 & & 0.15 & 0.11 & 0.07 & 0.05 & \\
\hline $\begin{array}{l}\text { Prevalence } \\
\text { chronic use }\end{array}$ & 18,170 & 8369 & 16,371 & 20,227 & 63,137 & 3578 & 2207 & 6737 & 7972 & 20,494 \\
\hline \multicolumn{11}{|l|}{ Privately insured } \\
\hline 2008 population & $1,717,063$ & $2,036,468$ & $10,526,582$ & $30,632,774$ & & $1,717,063$ & $2,036,468$ & $10,526,582$ & $30,632,774$ & \\
\hline $\begin{array}{l}\text { Chronic use } \\
\text { (\% of population) }\end{array}$ & 0.47 & 0.27 & 0.04 & 0.03 & & 0.12 & 0.08 & 0.02 & 0.01 & \\
\hline $\begin{array}{l}\text { Prevalence } \\
\text { chronic use }\end{array}$ & 8025 & 5474 & 3789 & 8595 & 25,883 & 2015 & 1643 & 1650 & 2420 & 7728 \\
\hline $\begin{array}{l}\text { Total with } \\
\text { chronic use }\end{array}$ & 26,195 & 13,843 & 20,160 & 28,822 & 89,020 & 5593 & 3850 & 8387 & 10,392 & 28,222 \\
\hline
\end{tabular}

${ }^{a}$ Due to rounding, the population counts multiplied by percentages do not exactly match the estimates

more restrictive definition of chronic metoclopramide use, over $90 \%$ also had concomitant dispensing of a gastric acid suppressor in both databases (data not shown).

Table 4 presents estimates of the total number of US children with chronic use of metoclopramide for each definition of chronic use by age group. Numbers were based on population estimates and the percentage of chronic users from each of the databases. Using the 35-day criterion for chronic use of metoclopramide, 89,020 US children used metoclopramide chronically. Of these, approximately $70 \%$ were Medicaid insured, and $45 \%$ were $<2$ years old. Using the 130-day criterion for chronic use of metoclopramide, 28,222 US children used metoclopramide chronically. Of these, approximately three-quarters were Medicaid insured and $33 \%$ were $<2$ years old.

\section{Discussion}

This study used two large healthcare claims databases representing privately insured and Medicaid populations, respectively, to calculate population-based estimates of chronic metoclopramide use in children aged 17 or less and describe the prevalence of diagnoses likely to be the reasons for this use. Chronic use required at least two dispensings. While it is possible they were filled but not used, taking the trouble and expense of filling a prescription twice suggests that the medication was used. Among this study's limitations was (1) the assumption that children who were not Medicaid insured were privately insured, effectively grouping any uninsured children with the privately insured. This resulted in a conservative estimate in the sense that privately insured children had lower rates of chronic use than did Medicaid insured children, but may have yielded an over-estimate to the extent that there were uninsured children who had no access to care. (2) Although it is unlikely for anyone to have private insurance and Medicaid insurance in the same 6-month period, there is no way to identify patients who may appear in both databases. (3) A diagnosis of GERD in the medical record at approximately the time that a child began chronic metoclopramide use, and a high prevalence of acid suppressive medication use among these children does not demonstrate that these children met the criteria for treatmentresistant GERD, though it strongly suggests that metoclopramide was being used to treat symptoms suggestive of GERD that did not respond adequately to more conventional treatment. An alternative explanation is that metoclopramide was used for symptoms of chronic non-GERD-related nausea, vomiting or delayed gastric emptying that may or may not have been associated with GERD, but for the patients with a diagnosis of GERD, there was a high prevalence of acid-suppressing medication use and reason to believe that some caregiver thought this was a credible diagnosis.

Chronic users were characterized according to three broad GI-related groups that might account for their use of metoclopramide: (1) a diagnosis of GERD, (2) GI diagnoses other than GERD, such as gastroparesis and vomiting, and (3) neuro-GI diagnoses other than GERD, but known to be associated with a high risk of chronic GERD, such as cerebral palsy. The largest subgroup of patients was those with a diagnosis of GERD, accounting for 52-87\% of chronic users regardless of use definition, depending on age. 
In both databases, the percentage of chronic users of metoclopramide who also had a diagnosis of GERD decreased from the youngest to the oldest age groups. There may be several explanations for this phenomenon. Higher chronic use in the youngest patients with a diagnosis of GERD may reflect an effort to avoid the need for anti-reflux surgery by using both acid suppression plus a prokinetic agent in addition to the standard adjustments in the method and type of feeding [6,7]. Older age groups may be less likely to use metoclopramide chronically because, either their symptoms may ameliorate with age, or adverse events including irritability and dystonic reactions may discourage chronic use, or GERD surgery, if successful, may make medications that treat reflux unnecessary.

\section{Conclusion}

This study indicates that, though other agents such as erythromycin, or, by special arrangements, cisapride or domperidone, are used for treatment of GI motility disorders [5, 12], between 28,222 and 89,020 US children aged 17 or younger chronically use metoclopramide even with its relatively high-risk adverse event profile. Chronic use of metoclopramide in children with a diagnosis of GERD is more prevalent in children aged $<1$ year and decreases in older age groups. However, a higher percentage of older patients have neurological impairment accompanying their GERD when they use chronic metoclopramide compared with younger children. The substantial amount of prescribing of metoclopramide for chronic use appears to reflect a perception that prokinetic agents may be useful when added to acid-suppressive treatment of some children with chronic symptoms consistent with refractory GERD, and a perception that there is a need for prokinetic drugs for this population.

Acknowledgments The authors thank Rupa Makadia (Epidemiology, Janssen Research and Development, LLC) for checking the programming code to help ensure accurate results.

Ethics committee waiver The New England Institutional Review Board (NEIRB\# 12-284) has determined that studies conducted in the databases used in this study do not meet the definition of human subjects research.

Funding/support The work was supported by Janssen Research and Development, LLC.

Role of the sponsor The sponsor approved the paper for submission for publication. Since all authors are employees of Janssen R \& D, in that sense, the sponsor had involvement in study design, the analysis and interpretation of the data and the writing of the manuscript.

Conflict of interest disclosures All authors are employed by Janssen Research and Development, LLC, which manufactures domperidone, a prokinetic that is not marketed in the USA.
Author contributions and accountability All authors made substantial contributions to and take full responsibility for the data acquisition and the integrity of the data analysis and interpretation. All authors are in agreement to be accountable for all aspects of the work and agree to allow the journal to review their data if requested. Each author listed on the manuscript has seen and approved the submission of this version of the manuscript.

Open Access This article is distributed under the terms of the Creative Commons Attribution-NonCommercial 4.0 International License (http://creativecommons.org/licenses/by-nc/4.0/), which permits any noncommercial use, distribution, and reproduction in any medium, provided you give appropriate credit to the original author(s) and the source, provide a link to the Creative Commons license, and indicate if changes were made.

\section{References}

1. Criag WR, Hanlon-Dearman A, Sinclair C et al. Metoclopramide, thickened feedings, and positioning for gastroesophageal reflux in children under two years. Cochrane Database Syst Rev 2004.

2. Hassall E. Decisions in diagnosing and managing chronic gastroesophageal reflux disease in children. $\mathrm{J}$ Pediatr. 2005;146:S3-12 (Doc ID:EDMS-ERI-71594676).

3. Jeurnink SM, van Herwaarden-Lindenboom MYA, Siersema PD, Fischer K, Houwen RHJ, van der Zee D. Barrett's esophagus in children: does it need more attention? Dig Liver Dis. 2011;43:682-7 (Doc ID:EDMS-ERI-71594670).

4. ANI Pharmaceuticals, Inc. 2011. Reglan ${ }^{\circledR}$ tablets (metoclopramide tablets USP). Retrieved from http://www.accessdata.fda. gov/drugsatfda_docs/label/2011/017854s058lbl.pdf. Accessed 21 Jan 2015.

5. Zanon, et al. Off-label prescribing patterns of antiemetics in children: a multicenter study in Italy. Eur J Pediatr. 2013;172(3):361-7.

6. Lasser MS, Liao JG, Burd RS. National trends in the use of antireflux procedures for children. Pediatrics. 2006;118:1828-35.

7. Vandenplas, et al. Pediatric Gastroesophageal Reflux Clinical Practice Guidelines: Joint Recommendations of the North American Society of Pediatric Gastroenterology, Hepatology, and Nutrition and the European Society of Pediatric Gastroenterology, Hepatology, and Nutrition. J Pediatr Gastroenterol Nutr. 2009;49:498-547.

8. Sullivan PB. Gastrointestinal problems in the neurologically impaired child. Balliere's Clin Gastroenterol. 1997;11:529-46.

9. Staiano A, et al. Disorders of oesophageal motility in children with psychomotor retardation and gastro-oesophageal reflux. Eur J Pediatr. 1991;150:638-41.

10. Centers for Medicare \& Medicaid Services. MSIS State Summary FY 2008. Table 05 Fiscal Year 2008 Medicaid Eligibles by Age Group. http://www.cms.gov/Research-Statistics-Data-and-System s/Computer-Data-and-Systems/MedicaidDataSourcesGenInfo/MS IS-Tables-Items/CMS1254739.html. Accessed 15 Jan 2014.

11. Table 1. Intercensal estimates of the resident population by sex and five-year age groups for the United States: April 1, 2000 to July 1, 2010 (US-EST00INT-01). Source: U.S. Census Bureau, Population Division. Release Date: Sept 2011.

12. Warner Chilcott, Inc. (2008). Erythromycin [package insert]. Rockaway, NJ: Warner Chilcott, Inc. 